\title{
Changes in structure and conduction type upon addition of Ir to $\mathrm{ZnO}$ thin films
}

\author{
M. Zubkins a , R. Kalendarev ${ }^{\text {a }}$, J. Gabrusenoks ${ }^{\text {a }}$, A. Plaude ${ }^{\text {a }}$, A. Zitolo ${ }^{\text {b }}$, A. Anspoks ${ }^{\text {a }}$, K. Pudzs ${ }^{\text {a }}$, K. Vilnis ${ }^{\text {a }}$, \\ A. Azens ${ }^{\text {a }}$, J. Purans ${ }^{\mathrm{a}}$ * \\ a Institute of Solid State Physics, University of Latvia, Kengaraga 8, LV-1063 Riga, Latvia \\ ${ }^{\mathrm{b}}$ Synchrotron SOLEIL, L'Orme des Merisiers, Saint-Aubin - BP 48, 91192 GIF-sur-YVETTE CEDEX, France
}

Keywords:

Amorphous doped $\mathrm{ZnO}$

Iridium

Thin films

Reactive DC magnetron co-sputtering

\begin{abstract}
A bstract
$\mathrm{Zn}-\mathrm{Ir}-\mathrm{O}(\mathrm{Zn} / \mathrm{Ir} \approx 1 / 1)$ thin films have been reported to be a potential p-type TCO material. It is, however, unknown whether it is possible to achieve p-type conductivity at low Ir content, and how the type and the magnitude of conductivity are affected by the film structure. To investigate the changes in properties taking place at low and moderate Ir content, this study focuses on the structure, electrical and optical properties of ZnO:Ir films with iridium concentration varying between 0.0 and 16.4 at.\%.

$\mathrm{ZnO}: \mathrm{Ir}$ thin films were deposited on glass, $\mathrm{Si}$, and $\mathrm{Ti}$ substrates by $\mathrm{DC}$ reactive magnetron co-sputtering at room temperature. Low Ir content (up to 5.1 at. \%) films contain both a nano-crystalline wurtzite-type ZnO phase and an X-ray amorphous phase. The size of the crystallites is below $10 \mathrm{~nm}$ and the lattice parameters a and $\mathrm{c}$ are larger than those of pure $\mathrm{ZnO}$ crystal. Structural investigation showed that the film's crystallinity declines with the iridium concentration and films become completely amorphous at iridium concentrations between 7.0 and 16.0 at.\% An intense Raman band at approximately $720 \mathrm{~cm}^{-1}$ appears upon Ir incorporation and can be ascribed to peroxide $\mathrm{O}_{2}^{2-}$ ions. Measurable electrical conductivity appears together with a complete disappearance of the wurtzitetype $\mathrm{ZnO}$ phase. The conduction type undergoes a transition from n- to p-type in the $\mathrm{Ir}$ concentration range between 12.4 and 16.4 at.\%. Absorption in the visible range increases linearly with the iridium concentration.
\end{abstract}

\section{Introduction}

Transparent conducting oxides (TCOs) are materials having high optical transparency and electrical conductivity simultaneously. These materials are important components for information (flat panel displays) and energy (thin film solar cells, low-emissivity windows) technologies [1,2]. Most TCO thin films, such as $\operatorname{In}_{2} \mathrm{O}_{3}: \mathrm{Sn}$ (ITO), $\mathrm{ZnO}$ :Al (AZO) and $\mathrm{SnO}_{2}: \mathrm{F}$, are n-type semiconductors [3]. The most studied ptype TCO materials are $\mathrm{Cu}$-based thin films with delafossite crystal structure $\left(\mathrm{AMO}_{2}\right)$ [4], but they suffer from low conductivity mainly because of low hole mobility [2] and indirect band gap [5], which is not optimal for optical devices. In addition, p-type conductivity is not retained in the amorphous phase, and an elevated substrate temperature is required for thin film deposition [3], which is not suitable for deposition on polymer substrates or in organic solar cell technologies.

Although doped $\mathrm{ZnO}$ thin films are promising n-type TCO materials $[6,7]$, obtaining p-type $\mathrm{ZnO}$ thin films would be an important milestone in transparent electronics, allowing the production of wide band gap $\mathrm{p}^{-}$ $\mathrm{n}$ homo-junctions $[8,9]$. P-type doping in $\mathrm{ZnO}$ thin films is hindered by a

\footnotetext{
* Corresponding author.

E-mail address: purans@cfi.lu.lv (J. Purans).
}

self-compensation effect from native donor defects $\left(\mathrm{V}_{\mathrm{O}}\right.$ and $\left.\mathrm{Zn}_{\mathrm{i}}\right)$ and/or hydrogen incorporation and mostly requires elevated growth temperatures [10]. The conductivity of p-type $\mathrm{ZnO}$ thin films is substantially lower compared to $\mathrm{n}$-type $\mathrm{ZnO}$. The reason for the lower conductivity is the large effective mass and thus the low mobility of the holes in the valence band, which is mainly composed of $p$ orbital levels of oxygen. A new approach to obtain p-type $\mathrm{ZnO}$ instead of doping is to produce a significant number of $\mathrm{Zn}$ vacancies and their complexes in order to generate p-type charge carriers $[11,12]$.

A relatively new and different class of p-type TCO is spinels $\mathrm{ZnM}_{2} \mathrm{O}_{4}$ ( $\mathrm{M}=\mathrm{Co}, \mathrm{Rh}, \mathrm{Ir}$ ) [13]. In $\mathrm{ZnM}_{2} \mathrm{O}_{4}$ spinel structure, $\mathrm{Zn}$ is bonded to four $\mathrm{O}$ atoms in a tetrahedral environment and $\mathrm{M}$ is bonded to six $\mathrm{O}$ atoms in an octahedral environment [14,15]. It has been reported that $\mathrm{ZnIr}_{2} \mathrm{O}_{4}$ polycrystalline thin films deposited without intentional doping are transparent $(60 \%$ at $550 \mathrm{~nm})$ p-type semiconductors $\left(\sigma=3.4 \mathrm{Scm}^{-1}\right.$, $\mathrm{E}_{\mathrm{g}}=2.97 \mathrm{eV}$ ) [16]. $\mathrm{ZnIr}_{2} \mathrm{O}_{4}$ is a thermodynamically metastable phase with cation vacancies and antisites having the lowest formation energies among the native defects in the disorder spinel structure [17]. These defects act like shallow acceptors and could be responsible for spontaneous p-type semiconducting $[15,17]$. It is significant that these materials retain p-type conductivity also in the amorphous phase [18, 19], however, in this case Ir ion more likely has the valence state of 
$4+$ instead of $3+[20]$. In the case of $\operatorname{Ir}(4+)$ oxide $\left(\mathrm{IrO}_{2}\right)$, absorption below $2.5 \mathrm{eV}$ is increased by $\mathrm{Ir} \mathrm{d}-\mathrm{d}$ and free electron absorption and above $3.0 \mathrm{eV}$ by $\mathrm{O}$ p-Ir d transitions [21].

Light ZnO doping with Ir has been studied by first principles DFT calculation using the B3LYP hybrid functional [22] with only one experimental investigation [23]. The theoretical studies show that the substitution defects of $\mathrm{Ir}_{\mathrm{n}}^{+2}$ and $\mathrm{Ir}_{\mathrm{Zn}}^{+3}$ create a series of localized states in the gap, and the transitions between them deteriorate the transmittance of the film. Although the formation energy of $\operatorname{Ir}_{\mathrm{Zn}}^{+3}$ favouring a hole localization on $\mathrm{Ir}$ is smaller than that of $\mathrm{Ir}_{\mathrm{Zn}}$, the formation energies of both types of defects are relatively large and the creation of these defects is unlikely [22]. However, the possibility of achieving p-type conductivity with light Ir doping is still unclear.

In the previous papers [24,25], the structural, electrical and optical properties of amorphous high Ir content $\mathrm{Zn}$-Ir-O thin films were investigated without covering the part with low iridium content. However, it is still unknown at what Ir concentration the originally crystalline $\mathrm{ZnO}$ structure becomes amorphous or whether there is a correlation between the film structure and the $\mathrm{n}$ - or p-type of conductivity. The present study focuses on the structure and the optical and electrical properties of $\mathrm{ZnO}$ :Ir thin films with low to moderate Ir concentrations. Room temperature deposition in this study was chosen in order to make the process compatible with low temperature technologies, such as those used for organic solar cells.

\section{Experimental details}

ZnO:Ir thin films were deposited on glass, $\mathrm{Si}$, and Ti substrates, by DC reactive magnetron co-sputtering from metallic $\mathrm{Zn}$ and Ir targets $(145 \mathrm{~mm} \times 92 \mathrm{~mm} \times 3 \mathrm{~mm})$ in an $\mathrm{Ar}+\mathrm{O}_{2}$ atmosphere (Fig. 1). The substrates were washed in acetone before deposition. The chamber was pumped to base pressure below $1 \times 10^{-5}$ Torr by a turbo-molecular pump. The substrate was not heated intentionally, the deposition pressure was $10 \mathrm{~m}$ Torr. The target to substrate separation was approximately $9 \mathrm{~cm}$. The $\mathrm{Zn}$ target was sputtered in a constant DC mode at a power of $200 \mathrm{~W}$. To vary the $\mathrm{Zn}$ to Ir ratio in the films, the Ir target was sputtered with the power varying between 6 and $70 \mathrm{~W}$ in a low frequency $(100 \mathrm{~Hz})$ pulse regime. The $\mathrm{Ar}$ and $\mathrm{O}_{2}$ gas flow rates were kept constant at $20 \mathrm{sccm}$ and $10 \mathrm{sccm}$, respectively. In general terms, the deposition atmosphere is thought to be sufficiently oxygen rich so as not to produce oxygen deficient films with potential defects promoting n-type conductivity. An additional semi-transparent metallic grid was placed above the Ir target in order to prevent some part of the sputtered Ir atoms from reaching the substrate and achieve low iridium concentrations, because the Ir sputtering rate under these conditions is noticeably higher than that of the $\mathrm{Zn}$. A set of samples was deposited with Ir concentrations in the films ranging from 0.0 to 16.4 at.\% (Fig. 2). It can be seen from Fig. 2 that the deposition rate and the Ir concentration vary

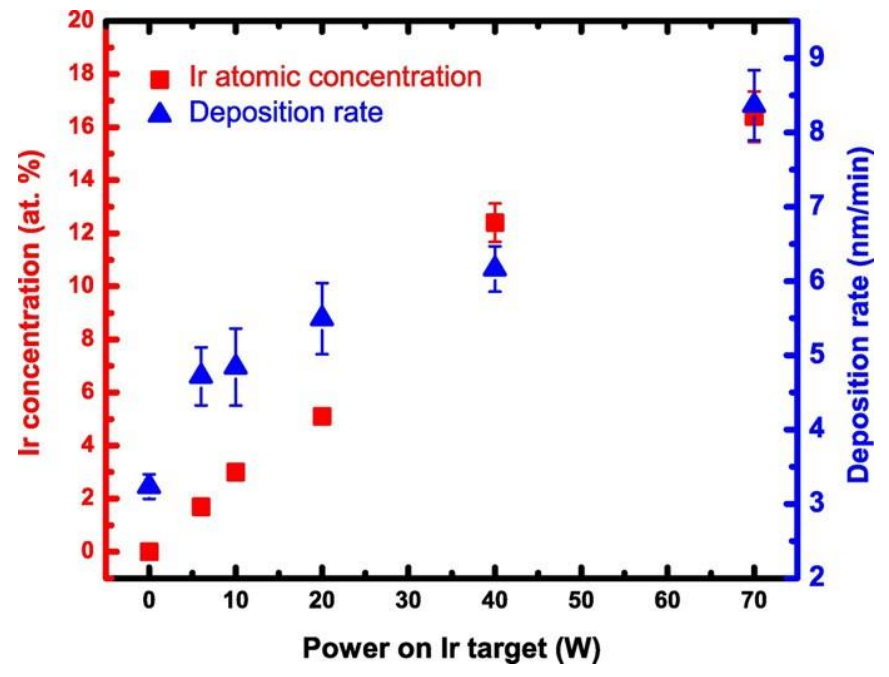

Fig. 2. Ir atomic concentration and deposition rate as a function of power on the Ir target.

in a close-to-linear fashion with the power on the Ir target, allowing for a simple and reliable way of depositing films with varied Ir contents.

The composition, structure, and the optical and electrical properties of the ZnO:Ir thin films were studied by X-ray fluorescence (XRF), X-ray diffraction (XRD), X-ray absorption (XRA), and Fourier transform infrared (FTIR) spectroscopy, as well as by two-beam optical spectrophotometry, profilometry, the Van der Pauw method, and Seebeck coefficient measurements. The film's thickness was determined by a profilometer, CART Veeco Dektak 150. The deposition rate of the film increases from 3.2 to $8.4 \mathrm{~nm} / \mathrm{min}$ (thickness from 388 to $1008 \mathrm{~nm}$ ) as the power on the iridium target increases from 0 to $70 \mathrm{~W}$ (Fig. 2).

An elemental analysis of the films was done by an X-ray fluorescence spectrometer, Eagle III. Due to the fact that it is difficult with XRF to quantify elements lighter than sodium, our measurements contain only the $\mathrm{Zn}$ and Ir atomic concentration ratio. The XRF spectra contained well detectable and well distinguished $\mathrm{Zn} \mathrm{Ka}$ and $\mathrm{Ir}$ La fluorescence lines. The quantification of the peak intensities was made by the spectrometer built-in calculations based on the fundamental-parametermodel. The ZnO:Ir films in the relevant thickness range are highly transparent to the X-rays, therefore the intensities of the fluorescence peaks are directly proportional to the films thickness. In the order to check the accuracy of the calculation results XRF spectra of pure $\mathrm{ZnO}$ thin films with varied thickness were measured as well. The obtained linear dependence of the $\mathrm{Zn} \mathrm{Ka}$ peak intensity on the thickness was used to recalculate the atomic concentration in the $\mathrm{ZnO}$ :Ir samples. The atomic concentrations obtained in both cases matched well within margin of error. The error bars for the Ir concentration in Fig. 2 reflect the variation

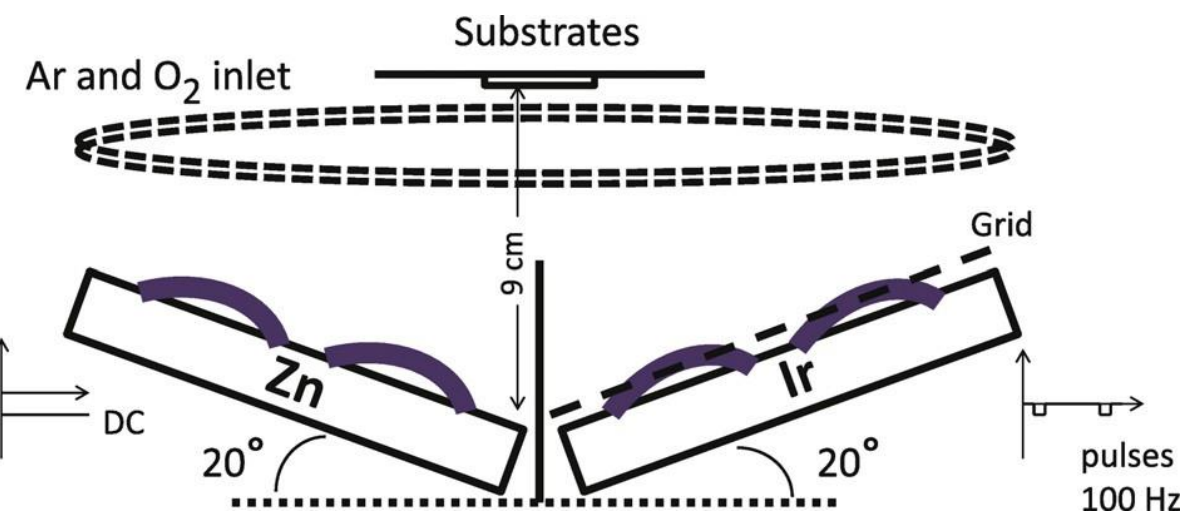

Fig. 1. Scheme of the reactive magnetron co-sputtering system. 
of the concentration within the analysed samples. This variation is most likely due to the two-magnetron configuration used for the deposition.

The structure was examined by an X-ray diffractometer with $\mathrm{Cu} \mathrm{Ka}$ radiation, PANalytical XPert Pro.

The Zn K-edge (9659 eV) and $\mathrm{Ir} \mathrm{L}_{3}$-edge $(11,215 \mathrm{eV}) \mathrm{X}$-ray absorption spectra (XAS) were measured in transmission mode at the SOLEIL synchrotron bending-magnet beamline Samba [26] in ambient conditions. The beamline is equipped with a sagittal focusing $\mathrm{Si}(220)$ monochromator and two Pd-coated collimating/focusing mirrors. The mirrors have been used to remove X-ray harmonics. The beam intensity was measured using three ionization chambers filled with argon and krypton gas. The samples were optimized by making a stack of thin films, simultaneously sputtered on polyimide tape, in order to achieve the best sample homogeneity and thickness. The deposition parameters of the samples for the XAS measurements can be found in [24].

The Fourier transform infrared (FTIR) absorbance spectra were measured by using a VERTEX 80v vacuum FTIR spectrometer. The experiments were performed in the range from 60 to $4000 \mathrm{~cm}^{-1}$, with the interferometer working in vacuum and with a resolution of $4 \mathrm{~cm}^{-1}$.

Raman scattering spectroscopy measurements were carried out at room temperature using a SPEX1403 monochromator with multichannel detectors and an inVia Renishaw Raman microscope. The experimental resolution was $5 \mathrm{~cm}^{-1}$. An Ar laser $(514.5 \mathrm{~nm})$ and YAG second harmonics laser $(532 \mathrm{~nm})$ were used as the excitation source. The incident beam power was about $5 \mathrm{~mW}$.

The film's transmittances, in the range from 200 to $1100 \mathrm{~nm}$, were determined by a double-beam spectrophotometer, Analytik Jena AG Specord 210. The electrical resistivity as well as the temperature dependence were measured in the Van der Pauw configuration by using the Hall effect system HMS5000.

In-plane Seebeck coefficient measurements were made by a self-assembled measurement system. Copper contact lines were thermally evaporated on the top of the films using shadow masks. The distance between the $\mathrm{Cu}$ lines was $2 \mathrm{~mm}$ and the length was $8 \mathrm{~mm}$. The temperature difference was measured with two K-type thermocouples pressed to the ends of the $\mathrm{Cu}$ lines. The temperature was controlled with two Peltier elements and a Stanford Research Systems Temperature PTC10 temperature controller. The Seebeck voltage was measured with a Keithley 2128 nanovoltmeter.

\section{Results and discussion}

\subsection{X-ray diffraction measurements (XRD)}

The XRD patterns of the films deposited on glass, recorded over a range for $2 \theta$ of $10970^{\circ}$, are shown in Fig. 3. A significant diffraction peak, which corresponds to the (002) plane, was observed at around $34^{\circ}$ for the pure and lightly doped $\mathrm{ZnO}$ films, indicating that the films contain a crystalline phase growing preferentially with the c-axis of the wurtzite-type $\mathrm{ZnO}(\mathrm{w}-\mathrm{ZnO}$ ) lattice perpendicular to the substrate surface. We note, however, that the intensity of the diffraction peak (002) is low, and comparable to the intensity of the halo peak around $23^{\circ}$ attributed to the glass substrate, which is indicative of the presence of a significant amorphous and/or nanocrystalline phase in the films. The low crystallinity even for the pure $\mathrm{ZnO}$ film is unexpected compared to the previous study [27], and could be due to the oblique angle deposition in a reactive atmosphere [28] and low deposition temperature. The films lose their crystalline phase orientation with increasing iridium concentration, and an additional peak of the (101) plane appears at 5.1 Ir at. \%. Further increase of the iridium concentration degrades the crystalline structure and the films become X-ray amorphous at $12.4 \mathrm{Ir}$ at.\%. No phases of $\mathrm{Zn}, \mathrm{Ir}, \mathrm{IrO}_{2}$ or $\mathrm{ZnIr}_{2} \mathrm{O}_{4}$ were detected. An earlier study showed that $\mathrm{Zn}-\mathrm{Ir}-\mathrm{O}$ thin films with Ir concentrations from 35 to 65 at. $\%$ are also X-ray amorphous [24].

In order to determine the diffraction angle and the full-width at halfmaximum (FWHM) of the asymmetric shape (002) peak, the peak was approximated by an asymmetric Gaussian function. The diffraction angle of the (002) peak shifts toward the lower angles and the FWHM increases with the iridium concentration.

The crystallite size in the [002] direction was calculated by the Scherrer equation and the size of the crystallites decreases from 7.5 to $5.0 \mathrm{~nm}$ with the iridium concentration (Fig. 4).

The lattice parameter c increases from 5.28 to $5.41 \AA$ as the iridium concentration increases from 0.0 to 5.1 at.\% (Fig. 4). The lattice parameter a calculated from the XRD pattern with the additional (101) peak for the $5.1 \mathrm{Ir}$ at.\% sample has a value of $3.30 \AA$. Both parameters, a and c, are greater than the standard $\mathrm{w}-\mathrm{ZnO}$ crystal lattice parameters $\mathrm{a}=3.25 \AA$ and $\mathrm{c}=5.21 \AA[29]$, indicating that incorporation of Ir in the lattice increases the unit cell of the crystal in all directions.

\subsection{X-ray absorption measurements (EXAFS)}

The Fourier transforms of the experimental $\mathrm{Zn} \mathrm{K}$-edge and $\mathrm{Ir} \mathrm{L}_{3}$-edge extended X-ray absorption fine structure spectra (FT-EXAFS-Zn,Ir) of $\mathrm{ZnO}$ :Ir thin films and reference compounds are shown in Fig. 5. The features in the FT-EXAFS correspond to the superposition of the various atom distribution functions for $\mathrm{Zn}$ and $\mathrm{Ir}$. But one should take into account that the amplitude and distances are distorted by photoelectron back scattering amplitudes and phase shifts [30].

The first feature of FT-EXAFS-Zn (Fig. 5a) at about $1.5 \AA$ originates from the first coordination shell of $\mathrm{Zn}$ - nearest oxygens. The second feature, at about $3 \AA$, comes mainly from the second coordination shell, here $\mathrm{Zn} \| \mathrm{Zn}$ (with real distance 3.2 and $3.25 \AA$ ). The feature at about $4.3 \AA$ originates from further $\mathrm{Zn} \| \mathrm{Zn}$ pairs $(4.57 \AA)$.

The FT-EXAFS-Zn spectra of the ZnO:Ir thin films are different from the polycrystalline $(\mathrm{c}-\mathrm{ZnO})$ reference spectrum. However, there are some similarities between $\mathrm{ZnO}: \mathrm{Ir}(7.0 \mathrm{Ir}$ at. \%) and the $\mathrm{c}-\mathrm{ZnO}$ reference up to the third coordination shell around $\mathrm{Zn}$. The FT-EXAFS- $\mathrm{Zn}$ feature for the first coordination shell is qualitatively the same, only an amplitude damping with an increase of Ir concentration happens, caused by increasing disorder in the $\mathrm{ZnllO}$ distances. Other features up to $4.5 \AA$ seen in c- $\mathrm{ZnO}$ are also present, but with significantly lower amplitudes, indicating increasing disorder. All the features above $4.5 \AA$ in the FTEXAFS-Zn signal of the ZnO:Ir(7.0 Irat.\%) are missing, due to significant structural disorder.

In the FT-EXAFS-Zn spectrum of the ZnO:Ir (16.4 Ir at.\%), even the feature around $2.9 \AA$, which corresponds to the second coordination shell, vanishes, indicating that the $\mathrm{ZnO}$ structure becomes completely amorphous for higher Ir concentrations.

Also in the FT-EXAFS-Ir spectra(Fig. 5b), all the features above $4.5 \AA$ are missing. However, features above $2.3^{\circ} \mathrm{A}$ correspond to the mix of the multiple scattering signal from the first coordination shell (Ir $\backslash$ OIO triangles) with some signal from the second coordination shell IrllMe (where Me can be $\mathrm{Zn}$ or Ir). This is different from the $\mathrm{Zn}$, where we cannot see any significant signal from the second coordination shell for higher Ir concentrations.

Therefore, from the EXAFS measurements, we can conclude that for small Ir concentrations (up to 7.0 Ir at.\%), the $\mathrm{ZnO}$ structure is nanocrystalline, but for higher Ir concentrations (e.g. $16.4 \mathrm{Ir}$ at.\%), the structure becomes completely amorphous.

\subsection{Vibrational analysis (FTIR and Raman measurements)}

The vibration properties of the $\mathrm{w}-\mathrm{ZnO}$ structure are characterized by the 9 optical phonons (irreducible representation $\Gamma_{\text {opt }}=A_{1}+2 B_{1}+E_{1}$ $+2 \mathrm{E}_{2}$, where the Emodes are twofold degenerate) [31]. Polarvibration modes $\mathrm{A}_{1}$ (along the $\mathrm{c}$ axis) and $\mathrm{E}_{1}$ (within the $\mathrm{a}-\mathrm{b}$ plane) are IR and Raman active and split into TO and LO modes due to macroscopic electric fields. The latter splitting (TO-LO) is larger than the $\mathrm{A}_{1}-\mathrm{E}_{1}$ splitting because the electric fields influence the vibration frequency more than the anisotropy of the bonds [32]. The $\mathrm{E}_{2}$ modes are only Raman active, and the $\mathrm{B}_{1}$ modes are silent. Both Fourier transform infra-red (FTIR) 


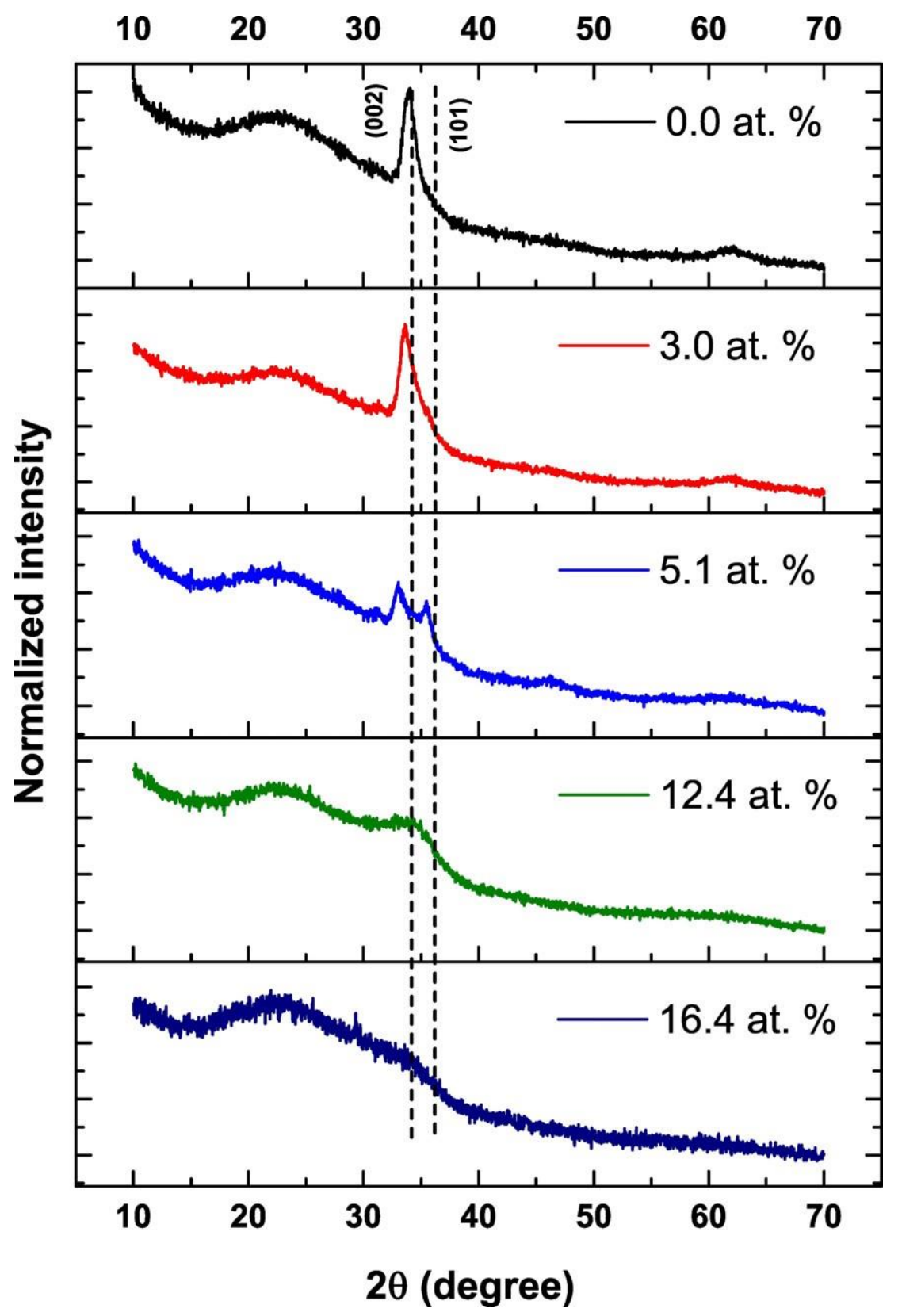

Fig. 3. XRD patterns of the $\mathrm{ZnO}$ :Ir thin films as a function of Ir atomic concentration.

and Raman spectroscopy were used to investigate the $\mathrm{ZnO}$ :Ir vibrational properties. A Lorentzian peak function was used to obtain the position (frequency of the mode) and full-width at half-maximum (FWHM) of the peaks.

In the IR absorption measurements, the films on Si substrates were used and the absorbance was normalized for the thickness. The samples were irradiated perpendicularly to the film's surface with non-polarised light. Only the transverse optical mode $\mathrm{E}_{1}^{\mathrm{TO}}$ around $410 \mathrm{~cm}^{-1}$ was observed (Fig. 6a) because the $\mathrm{ZnO}$ nano-crystallites are c-axis oriented. The frequency and FWHM of the peak of the transverse optical mode does not change significantly up to 5.1 at.\% of Ir (Fig. 6c). Above this value, the peak position shifts sharply toward higher wavenumbers, around $460 \mathrm{~cm}^{-1}$, and becomes wider. Above 5.1 at. $\%$ of Ir, the film's structure changes significantly, with the nano-crystallites becoming smaller and the structural atomic network gradually becoming different from crystalline $\mathrm{w}-\mathrm{ZnO}$ until the wurtzite phase becomes undetectable at $12.4 \mathrm{Ir}$ at.\%. The integrated intensity (peak area) decreases up to 9.5 at. \% at first and then starts to increase slightly above $12.4 \mathrm{Ir}$ at.\%.
The additional absorption peak around $200 \mathrm{~cm}^{-1}$ at higher Ir concentrations also indicates a new structural phase. A similar change of the structure is observed for $\mathrm{ZnO}$ :Ir samples deposited at $573 \mathrm{~K}$ as well, with the $\mathrm{w}-\mathrm{ZnO}$ structure being detectable for up to $20.0 \mathrm{Ir}$ at. $\%$ and not detectable for higher Ir concentrations.

Raman spectroscopy measurements were performed on the films deposited on the Ti substrates. The intensity of the Raman spectra was recalculated, taking into account the film's thickness and the absorption coefficient (see the section on the optical properties). In the Raman spectrum of the pure $\mathrm{ZnO}$ film, only $\mathrm{A}_{1}, \mathrm{E}_{2}$ and $\mathrm{E}_{2}$ bands (573, 437 and $97 \mathrm{~cm}^{-1}$, respectively) were detected (Fig. 6b) due to the experimental configuration and film orientation $(z(x+y, x+y) z)$. The band around $860 \mathrm{~cm}^{-1}$ originates from the $\mathrm{ZnO}_{2}$ phase [33] and the wide band around $1100 \mathrm{~cm}^{-1}$ from the second order mode $2 \mathrm{~A}_{1}^{\mathrm{LO}}$.

The Raman intensity for the pure $\mathrm{ZnO}$ films is relatively low compared to the new vibration mode around $720 \mathrm{~cm}^{-1}$ which appears after Ir incorporation (Fig. 6b) and was for the first time observed in Ref. [25]. However, signs of the $\mathrm{A}_{1}^{\mathrm{LO}}$ and $\mathrm{E}^{\text {gigh }}$ bands are still observable 


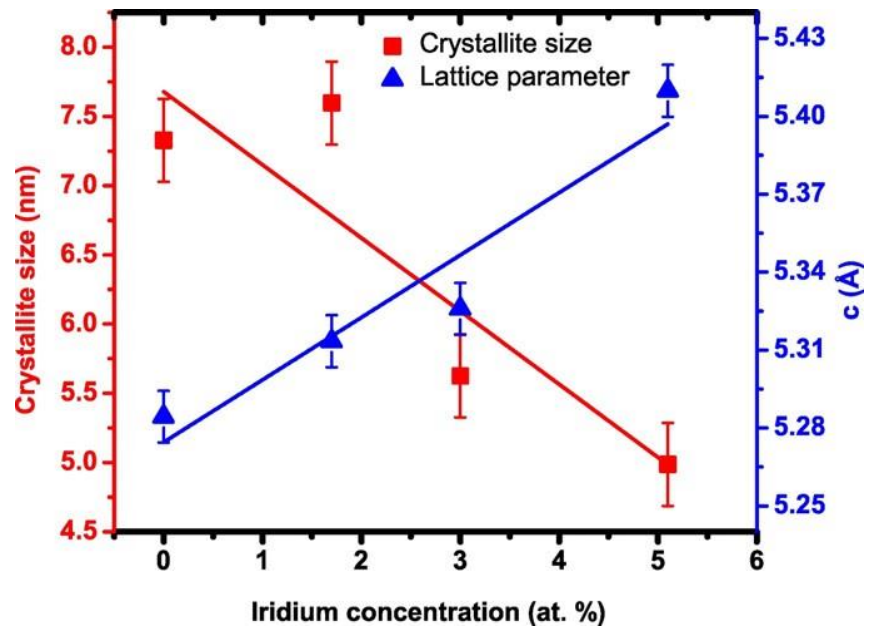

Fig. 4. Crystallite size and lattice parameter $\mathrm{c}$ as a function of Ir atomic concentration.

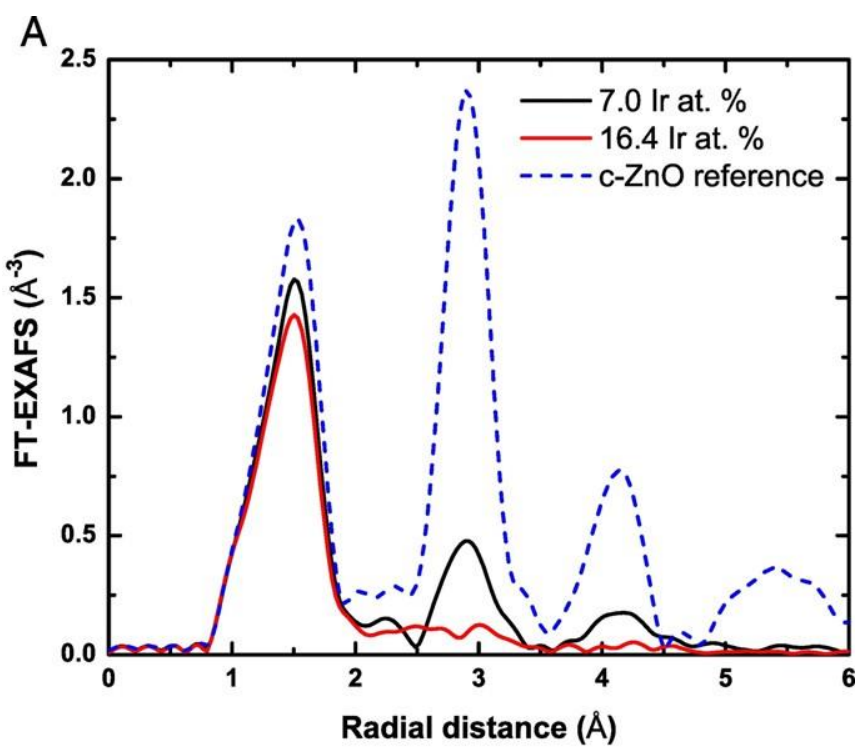

B

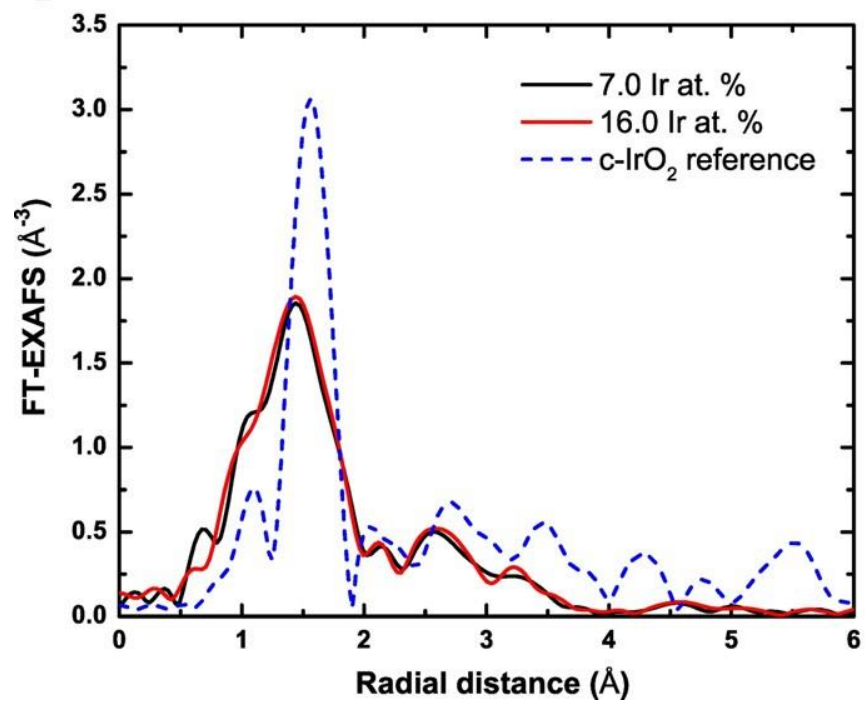

Fig. 5. Modulus of the FT-EXAFS spectra at Zn K-edge (a) and Ir $\mathrm{L}_{3}$-edge (b) for ZnO:Ir (7.0 and $16.0 \mathrm{Ir}$ at.\%) thin films and reference compounds - polycrystalline bulk c-ZnO and c$\mathrm{IrO}_{2}$. (Note: distances in the FT-EXAFS do not correspond to the real distances, due to the phase shifts of the signal.) up to 5.1 at.\% of Ir. A sharp decrease of the Raman intensity of the w- $\mathrm{ZnO}$ active modes was also observed in Ref. [34-36] after doping with transition metals. There is no clear evidence about the origin of the intense $720 \mathrm{~cm}^{-1}$ band. However, the present results do not support the assumption that the band arises from the $\mathrm{IrO}_{2}$ phase [23]. If the $\mathrm{IrO}_{2}$ phase were present, there should be an even more intense $\mathrm{E}_{\mathrm{g}}$ band around $560 \mathrm{~cm}^{-1}$ [37]. Theoretically, the $\mathrm{E}_{\mathrm{g}}$ band is forbidden in the backscattering configuration if the crystallites are oriented in the [001] direction [38], which is unlikely in this case due to the disordered structure. We performed Raman measurements under oblique angle to check whether oriented $\mathrm{IrO}_{2}$ crystallites are responsible for the $720 \mathrm{~cm}^{-1}$ band, but the obtained spectra (not presented here) did not exhibit any changes.

The frequency, the FWHM, and the integrated intensity of the intense $720 \mathrm{~cm}^{-1}$ band, are shown in Fig. $6 \mathrm{~d}$. The frequency shifts close to exponentially from 740 to $710 \mathrm{~cm}^{-1}$ as the Ir concentration grows from 1.7 to 16.4 at. $\%$. The FWHM undergoes a rapid step-like decrease from 120 to $90 \mathrm{~cm}^{-1}$ in the Ir concentration range from 5.1 to 9.5 at.\%. The behaviour of the frequency and the FWHM indicates that the change of the structure taking place upon an increase of Ir concentration up to 9.5 at.\% causes the relaxation of the vibration mode. The integrated intensity increases up to 5.1 at.\% at first and starts to decrease above 9.5 Ir at.\%. This is one more piece of evidence that the band does not originate from the $\mathrm{IrO}_{2}$ phase, because in that case, and also taking into account that the Raman intensity is directly proportional to the concentration of the active species, the intensity should have increased with the Ir concentration. The band around $1400 \mathrm{~cm}^{-1}$ is the second order mode of the $720 \mathrm{~cm}^{-1}$ band and behaves the same way with an increase in the Ir concentration. Additionally, a low intensity band around $230 \mathrm{~cm}^{-1}$ appears after Ir incorporation into the $\mathrm{ZnO}$.

It can be suggested that the $720 \mathrm{~cm}^{-1}$ band which is not IR active is caused by the presence of peroxide ions, and the band may be attributed to the stretching mode of the $\mathrm{O}_{2}$. Since the pure $\mathrm{ZnO}$ spectrum does not exhibit a $720 \mathrm{~cm}^{-1}$ band, the presence of Ir causes structural changes favourable for the generation of $\mathrm{Q}_{2}^{2-}$ with the OlO distance appropriate for the $720 \mathrm{~cm}^{-1}$ stretching frequency. Crystalline $\mathrm{ZnO}_{2}$ with an OllO bond length of approximately $1.5 \AA$ [39] has the characteristic Raman band around $840 \mathrm{~cm}^{-1}$ [33], while the theoretical studies on $\mathrm{ZnO}_{2}$ molecules show that the $\mathrm{O}_{2}$ stretching frequency can be as low as $682 \mathrm{~cm}^{-1}$ with an OlO bond length of $1.7 \AA$ [40]. For the stretching frequency of $720 \mathrm{~cm}^{-1}$, the appropriate OlO distance should be between 1.5 and $1.7 \AA$. We note that an abnormal Raman band around $670 \mathrm{~cm}^{-1}$ in the $\mathrm{Ce}^{3+}$ and $\mathrm{Tb}^{3+}$ doped $\mathrm{ZnO}$ was also observed in Refs. $[34,36]$, without any interpretation of the band's origin.

The framework of the $\mathrm{O}_{2}^{2-}$ in the nanoporous oxide $\mathrm{C} 12 \mathrm{~A} 7$ $\left(12 \mathrm{CaO} \cdot 7 \mathrm{Al}_{2} \mathrm{O}_{3}\right)$ with the frequency around $770 \mathrm{~cm}^{-1}$ is deeply studied in Ref. $\left[4{ }_{2}, 42\right]$. A high partial pressure of oxygen favours the formation of the $\mathrm{O}_{2}$, which is accomplished by a diffusion of $\mathrm{O}_{2}$ molecules from the atmosphere into nanocages of $\mathrm{C} 12 \mathrm{~A} 7$, where they react with $\mathrm{O}^{2-}$ radicals. Following this approach, the molecular oxygen used in the deposition process of the $\mathrm{ZnO}$ :Ir films could be caged in the nanopores and produce the peroxide ions $\mathrm{O}_{2}$. Our previous study [25] reveals that an $\mathrm{ZnO}: \mathrm{Ir}$ film has a porous structure.

\subsection{Electrical properties}

We studied the electrical transport of the thin films by measuring the DC electrical conductivity at room temperature and as a function of temperature between $90 \mathrm{~K}$ and $330 \mathrm{~K}$.

The resistivity of the films with low iridium atomic concentration (0.0-9.5 at.\%) is extremely high and exceeds the measurable range. The high oxygen content used in the sputtering ambient was obviously sufficient to prevent the formation of oxygen vacancies or zinc interstitials, which are native donors in the $\mathrm{ZnO}$ and the origin of spontaneous n-type conductivity [10]. However, the iridium impurities at low concentrations do not appear to have generated sufficient levels of defects 
A
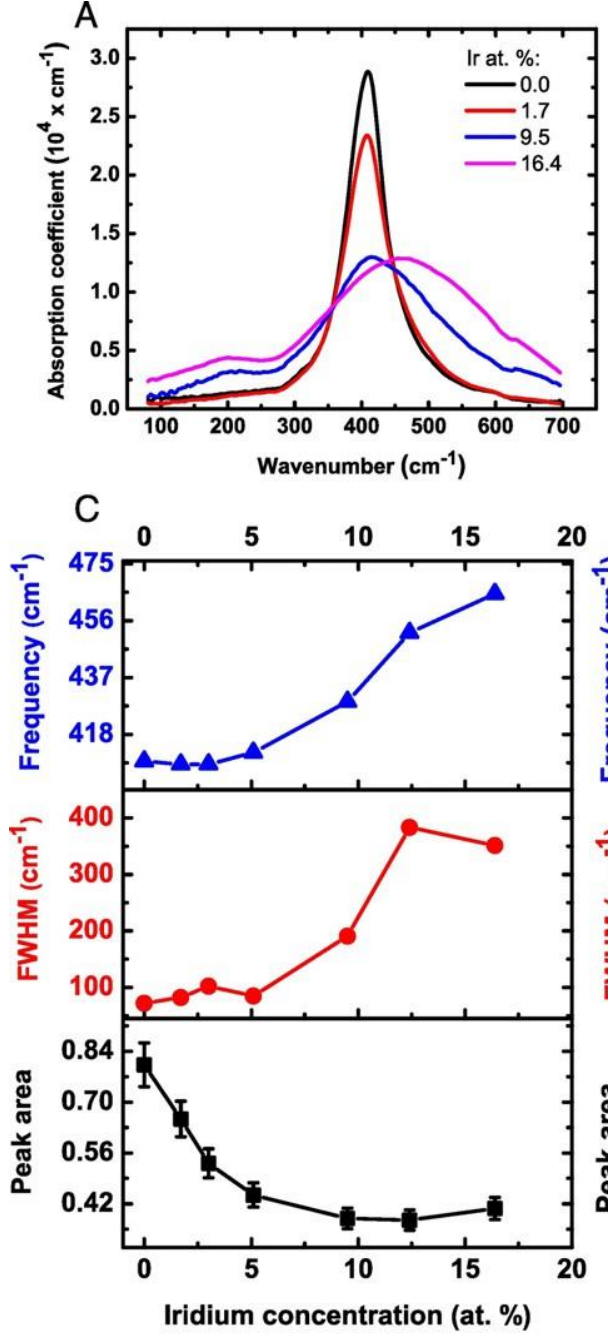

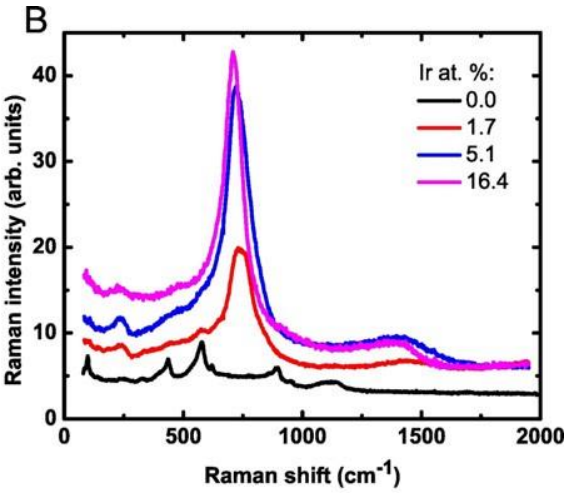

D

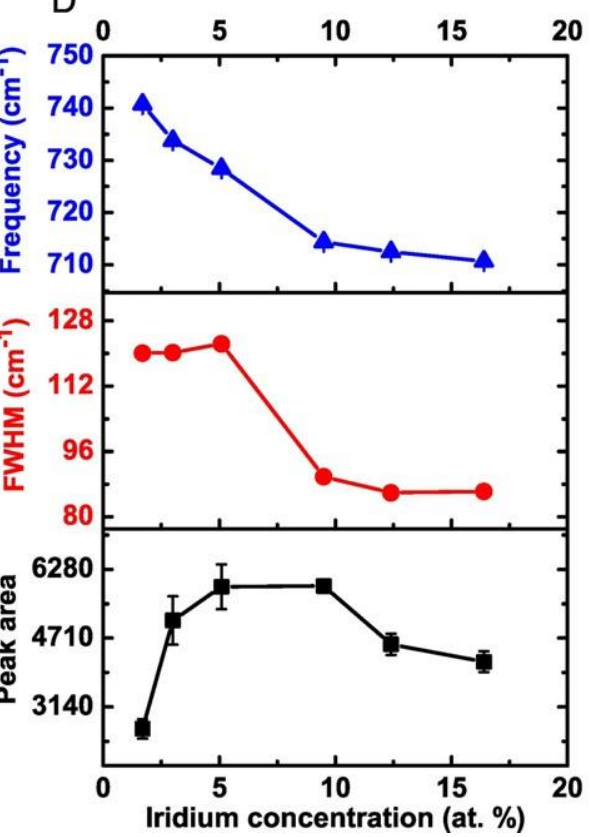

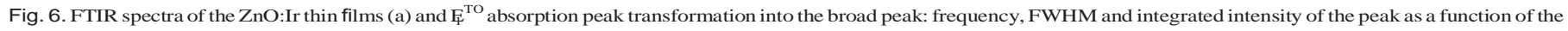

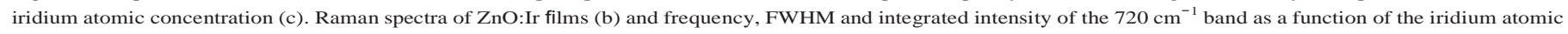
concentration (d).

to increase the conductivity significantly. A measurable resistivity of 83 $\Omega \mathrm{cm}$ appears at 12.4 at.\% of iridium, which correlates with the $\mathrm{w}-\mathrm{ZnO}$ structure disappearing completely according to the structural and vibrational investigation. For the $\mathrm{ZnO}$ :Ir films deposited at $573 \mathrm{~K}$, a measurable resistivity of $0.12 \Omega \mathrm{cm}$ appears at $19.1 \mathrm{Ir}$ at.\%, which again correlates with the fact that in this case the $\mathrm{w}-\mathrm{ZnO}$ structure becomes undetectable for Ir concentrations above 20.0 at.\%.

Hall effect measurements were non-reproducible, having a large variation in the Hall voltage, leading either to n- or p-type conductivity. It is known that Hall effect measurements for disordered and heterogeneous semiconductors are problematic and still under investigation [43, 44]. In addition, we note that there is no pronounced plasmon peak of the free electrons observed in the IR spectra of the films.

An Arrhenius plot of $\ln (\rho)$ against $1000 / \mathrm{T}\left(\mathrm{K}^{-1}\right)$ is shown in Fig. 7a. From the slope in Fig. 7a, thermally activated $\left(E_{a}=141 \mathrm{meV}\right)$ charge transport was found for the conducting sample with $12.4 \mathrm{Ir}$ at.\%, indicating a semiconductor type behaviour. However, the effect of temperature on resistivity of the next sample with $16.4 \mathrm{Ir}$ at.\% is insignificant $\left(\mathrm{E}_{\mathrm{a}}=\right.$ $14 \mathrm{meV}$ ). The experimental data were approximated by the function $\ln (\rho)=\mathrm{a}+\mathrm{bT}^{\xi}$, where $\mathrm{a}, \mathrm{b}$ and $\xi$ are fitting parameters, to determine the conduction model. Our data yields the value of $\xi=0.263$ (see Fig. 7a) for the sample with $12.4 \mathrm{Ir}$ at.\%, pointing at Mott's thermally activated variable range hopping conduction mechanism [45] in the studied film. We note that an almost constant resistivity, i.e., non- thermallyactivated conductivity, was found for the films with Ir content between 45 and 100 at.\% Ir [25], suggesting that the conductivity mechanism and Fermi level location undergoes a change as the Ir content increases.

The dependence of the Seebeck voltage on temperature was measured by varying temperature difference from -5 to $+5 \mathrm{~K}$. The average temperature was kept constant at $308 \mathrm{~K}$. The Seebeck coefficient was calculated as a slope coefficient of the obtained relations (see Fig. 7b). The larger data dispersion for the sample with $12.4 \mathrm{Ir}$ at. $\%$ is due to lower conductivity. The sign of the Seebeck coefficient changes from negative to positive (from -13.1 to $+6.8 \mu \mathrm{V} / \mathrm{K}$ ) if the iridium concentration increases from 12.4 to 16.4 at. $\%$. There is no clear interpretation of what causes the transition from n-type to p-type conductivity. The p-type conductivity could be accomplished through the amorphous $\mathrm{ZnO}$ matrix with $\mathrm{Zn}$ vacancies or through the complexes containing Ir. At a critical Ir concentration, a conduction path is obtained, and charge carriers can percolate through the material. However, the transition of the conduction type indicates that there are several competing conductivity mechanisms.

\subsection{Optical properties}

The transmittance of the $\mathrm{ZnO}$ :Ir films on glass was measured in the range from 200 to $1100 \mathrm{~nm}$. The absorption coefficient at $2.25 \mathrm{eV}$ 

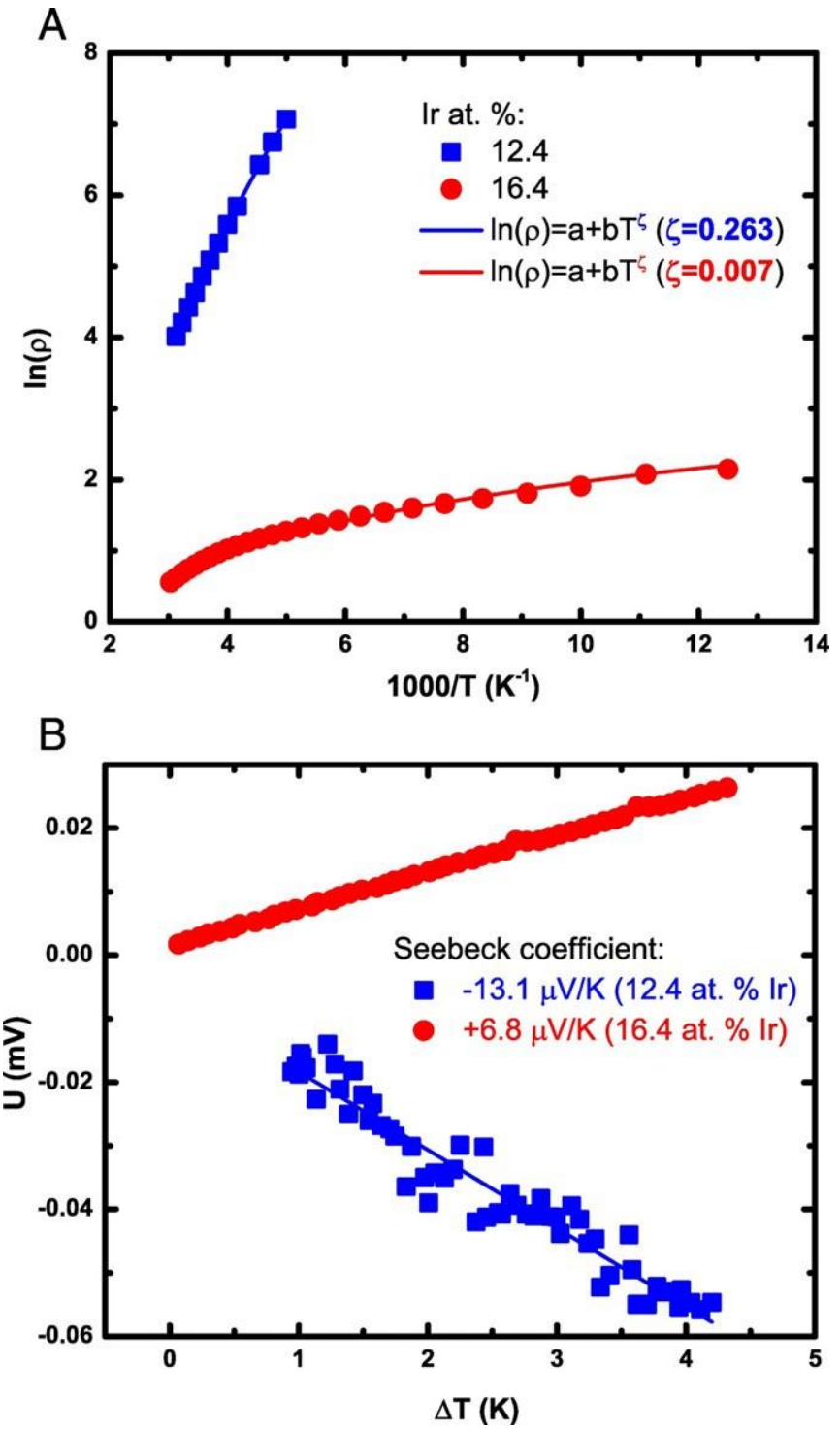

Fig. 7. Arrhenius plot $\ln (\rho)$ vs. $1000 / \mathrm{T}$ of the $\mathrm{ZnO}: \operatorname{Ir}(12.4$ and 16.4 at. $\%)$ thin films (a) and change of the Seebeck coefficient sign from negative to positive when Ir concentration changes from 12.4 to 16.4 at. $\%$.

$(550 \mathrm{~nm})$ increases linearly if the concentration of the Ir grows (Fig. 8a), indicating that transitions of the Ir containing phase is responsible for the reduced transmittance.

The optical band gap $\left(\mathrm{E}_{\mathrm{g}}\right)$ was estimated from the fundamental absorption edge by applying the Tauc model [46]. The optical band gap of the $\mathrm{ZnO}$ :Ir thin films does not shift with the iridium concentration up to 5 at.\% Ir and remains around $3.3 \mathrm{eV}$, which is the standard value for $\mathrm{ZnO}$ thin films (Fig. 8b). However, the sharpness of the absorption edge decreases. It is worth mentioning that there is no blue shift of the optical band gap together with the $\mathrm{ZnO}$ amorphization, as was reported in Refs. $[47,48]$. Furthermore, the $\mathrm{ZnO}_{2}$ phase detected in the pure $\mathrm{ZnO}$ Raman spectrum should be at low concentration, because $\mathrm{ZnO}_{2}$ exhibits a larger band gap than $\mathrm{ZnO}$ [49].

\section{Conclusions}

Reactive magnetron co-sputtering deposition from two targets is shown to be a suitable technique to dope thin films very accurately by using the sputtering power as the composition control parameter.

$\mathrm{ZnO}$ :Ir thin films with Ir concentrations in the range from 0.0 to 5.1 at.\% contain both nano-crystallites of $\mathrm{w}-\mathrm{ZnO}$ structure and an $\mathrm{X}$ -
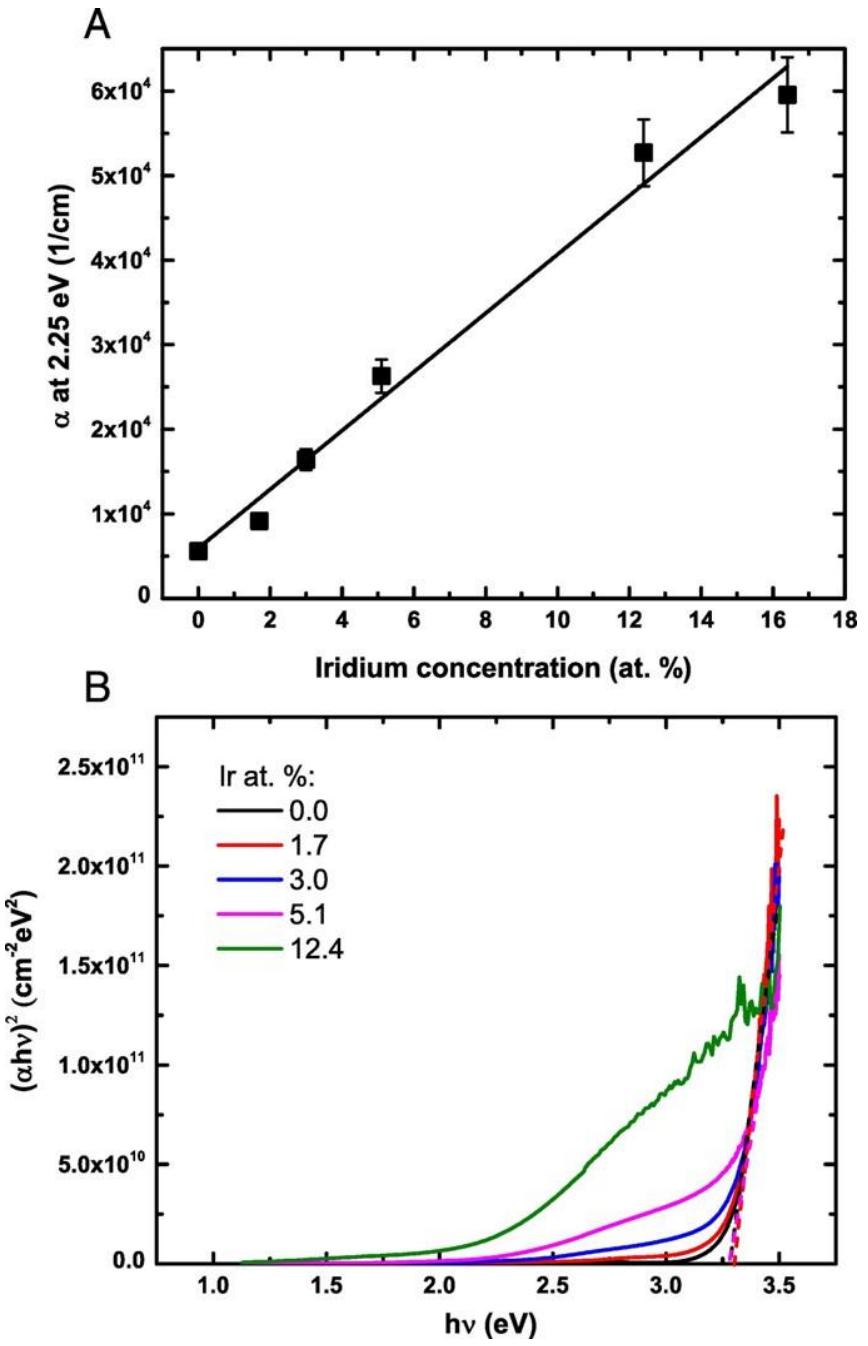

Fig. 8. Absorption coefficient of the $\mathrm{ZnO}$ : Ir thin films at $2.25 \mathrm{eV}$ as a function of iridium concentration (a). ( $(\mathrm{h} v)^{2}$ versus hv plot to measure the optical band gaps (b).

ray amorphous phase. The structure undergoes a transition from a wurtzite type structure to a structural atomic network different from crystalline $\mathrm{w}-\mathrm{ZnO}$ in the Ir concentration range from 5.1 to 12.4 at.\%, according to the Fourier transform infrared spectroscopy measurements. The structure becomes completely amorphous at an Ir concentration between 7.0 and 16.0 at.\%, according to the X-ray absorption spectra. After Ir incorporation, the $\mathrm{ZnO}$ structure contains peroxide $\mathrm{O}_{2}$ ions that are responsible for the intense Raman band around $720 \mathrm{~cm}^{-1}$.

$\mathrm{ZnO}:$ Ir thin films with an iridium concentration in the range from 0.0 to 9.5 at.\% are insulators. A measurable resistivity of $83 \Omega \mathrm{cm}$ at 12.4 at.\% of the iridium appears when the $\mathrm{w}-\mathrm{ZnO}$ structure disappears completely. The resistivity decreases even further at $16.4 \mathrm{Ir}$ at. $\%: 2.1 \Omega \mathrm{cm}$. The electrical transport changes from thermally activated hopping to metallic-like conductivity, and the conduction type undergoes a transition from n-type to p-type at an Ir concentration between 12.4 and 16.4 at.\%.

Absorption in the visible light band increases linearly with the iridium atomic concentration. The optical band gap of the films does not shift with the iridium concentration. However, the sharpness of the absorption edge decreases.

\section{Acknowledgements}

This work has been performed within the VMTKC project 18, agreement No. 1.2.1.1/16/A/005. 


\section{References}

[1] K. Ellmer, Past achievements and future challenges in the development of optically transparent electrodes, Nat. Photonics 6 (12) (2012) 809-817.

[2] L. Castañeda, Present status of the development and application of transparent conductors oxide thin solid films, Mater. Sci. Appl. 2 (9) (2011) 1233-1242.

[3] E. Fortunato, D. Ginley, H. Hosono, D.C. Paine, Transparent conducting oxides for photovoltaics, MRS Bull. 32 (03) (2007) 242-247.

[4] H. Kawazoe, M. Yasukawa, H. Hyodo, M. Kurita, H. Yanagi, H. Hosono, P-type electrical conduction in transparent thin films of $\mathrm{CuAlO}_{2}$, Nature 389 (6654) (1997) 939-942.

[5] D.O. Scanlon, K.G. Godinho, B.J. Morgan, G.W. Watson, Understanding conductivity anomalies in CuI-based delafossite transparent conducting oxides: theoretical insights, J. Chem. Phys. 132 (2) (2010), 024707.

[6] K.H. Kim, K.C. Park, D.Y. Ma, Structural, electrical and optical properties of aluminum doped zinc oxide films prepared by radio frequency magnetron sputtering, J. Appl. Phys. 81 (12) (1997) 7764-7772.

[7] V. Assuncao, E. Fortunato, A. Marques, H. Aguas, I. Ferreira, M. Costa, R. Martins, In fluence of the deposition pressure on the properties of transparent and conductive $\mathrm{ZnO}$ : Ga thin-film produced by rf sputtering at room temperature, Thin Solid Films 427 (1) (2003) 401-405.

[8] S. Chu, J. Lim, L. Mandalapu, Z. Yang, L. Li, J. Liu, Sb-doped p-Zn O/Ga-doped n-Zn O homojunction ultraviolet light emitting diodes, Appl. Phys. Lett. 92 (15) (2008) 152103.

[9] M. Joseph, H. Tabata, T. Kawai, P-type electrical conduction in ZnO thin films by Ga and N codoping, Jpn. J. Appl. Phys. 38 (11A) (1999) L1205.

[10] J.C. Fan, K. Sreekanth, Z. Xie, S. Chang, K.V. Rao, P-type ZnO materials: theory, growth, properties and devices, Prog. Mater. Sci. 58 (6) (2013) 874-985.

[11] L. Pan, S. Wang, W. Mi, J. Song, J.-J. Zou, L. Wang, X. Zhang, Undoped ZnO abundant with metal vacancies, Nano Energy 9 (2014) 71-79.

[12] A. Renaud, L. Cario, X. Rocquelfelte, P. Deniard, E. Gautron, E. Faulques, T. Das, F. Cheviré, F. Tessier, S. Jobic, Unravelling the origin of the giant $\mathrm{Zn}$ deficiency in wurtzite type $\mathrm{ZnO}$ nanoparticles, Sci Rep 5 (2015) 12914.

[13] H. Mizoguchi, M. Hirano, S. Fujitsu, T. Takeuchi, K. Ueda, H. Hosono, ZnRh2O4: A ptype semiconducting oxide with a valence band composed of a low spin state of Rh3+ in a 4 d6 configuration, Appl. Phys. Lett. 80 (7) (2002) 1207-1209.

[14] D.O. Scanlon, G.W. Watson, Band gap anomalies of the ZnM 2 III O 4 (M III $=$ Co, Rh, Ir) spinels, Phys. Chem. Chem. Phys. 13 (20) (2011) 9667-9675.

[15] D.M. Ramo, P.D. Bristowe, The effect of defects and disorder on the electronic properties of ZnIr2O4, J. Chem. Phys. 141 (8) (2014), 084704.

[16] M. Dekkers, G. Rijnders, D.H. Blank, ZnIr2O4, a p-type transparent oxide semiconductor in the class of spinel zinc-d6-transition metal oxide, Appl. Phys. Lett. 90 (2) (2007) 21903.

[17] M. Amini, H. Dixit, R. Saniz, D. Lamoen, B. Partoens, The origin of p-type conductivity in $\mathrm{ZnM} 2 \mathrm{O} 4(\mathrm{M}=\mathrm{Co}, \mathrm{Rh}, \mathrm{Ir})$ spinels, Phys. Chem. Chem. Phys. 16 (6) (2014) 2588-2596.

[18] S. Narushima, H. Mizoguchi, K.-i. Shimizu, K. Ueda, H. Ohta, M. Hirano, T. Kamiya, H. Hosono, A p-type amorphous oxide semiconductor and room temperature fabrication of amorphous oxide $\mathrm{p}^{-n}$ heterojunction diodes, Adv. Mater. 15 (17) (2003) 1409-1413.

[19] J.M. Dekkers, Transparent Conducting Oxides on Polymeric Substrates by Pulsed Laser Deposition, (Ph.D. thesis) University of Twente, Enschede, Netherlands, 2007.

[20] D.M. Ramo, P.D. Bristowe, Impact of amorphization on the electronic properties of Zn-Ir-O systems, J. Phys. Condens. Matter 28 (34) (2016) 345502.

[21] A.K. Goel, G. Skorinko, F.H. Pollak, Optical properties of single-crystal rutile Ru O 2 and Ir O 2 in the range 0.5 to $9.5 \mathrm{eV}$, Phys. Rev. B 24 (12) (1981) 7342.

[22] D.M. Ramo, P.D. Bristowe, Hybrid density functional calculations of the defect properties of ZnO: Rh and ZnO: Ir, Thin Solid Films 555 (2014) 112-116.

[23] G. Michail, V. Kambylafka, I. Kortidis, K. Tsagaraki, M. Androulidaki, G. Kiriakidis, V. Binas, M. Modreanu, E. Aperathitis, On the growth of transparent conductive oxide ternary alloys $\mathrm{Zn}-\mathrm{Ir}-\mathrm{O}$ (ZIRO) by the means of rf magnetron co-sputtering, Thin Solid Films 617 (2016) 3-8.

[24] M. Zubkins, R. Kalendarev, J. Gabrusenoks, K. Vilnis, A. Azens, J. Purans, Structural, electrical and optical properties of zinc-iridium oxide thin films deposited by DC reactive magnetron sputtering, Phys. Status Solidi C 11 (9-10) (2014) 1493-1496.

[25] M. Zubkins, R. Kalendarev, J. Gabrusenoks, K. Smits, K. Kundzins, K. Vilnis, A. Azens, J. Purans, Raman, electron microscopy and electrical transport studies of X-ray amorphous $\mathrm{Zn}$-Ir-O thin films deposited by reactive DC magnetron sputtering, IOP
Conference Series: Materials Science and Engineering, vol. 77, IOP Publishing 2015 , p. 012035

[26] V. Briois, E. Fonda, S. Belin, L. Barthe, C. La Fontaine, F. Langlois, M. Ribbens, F. Villain, SAMBA: The 4-40 keV X-ray Absorption Spectroscopy Beamline at SOLEIL, EDP Sciences, 2011 41-47.

[27] M. Zubkins, R. Kalendarev, K. Vilnis, A. Azens, J. Purans, Structural, electrical and optical characteristics of Al-doped zinc oxide thin films deposited by reactive magnetron sputtering, IOP Conference Series: Materials Science and Engineering, vol. 49, IOP Publishing 2013, p. 012057.

[28] Y.E. Lee, S.G. Kim, Y.J. Kim, H.J. Kim, Effect of oblique sputtering on microstructural modification of ZnO thin films, J. Vac. Sci. Technol. A 15 (3) (1997) 1194-1199.

[29] Ü. Özgür, Y.I. Alivov, C. Liu, A. Teke, M. Reshchikov, S. Doğan, V. Avrutin, S.-J. Cho, H. Morkoc, A comprehensive review of $\mathrm{ZnO}$ materials and devices, Journal of applied physics, 98 (4) (2005) 041301.

[30] V. Aksenov, M. Kovalchuk, A.Y. Kuzmin, Y. Purans, S. Tyutyunnikov, Development of methods of EXAFS spectroscopy on synchrotron radiation beams: review, Crystallogr. Rep. 51 (6) (2006) 908-935.

[31] T.C. Damen, S. Porto, B. Tell, Raman effect in zinc oxide, Phys. Rev. 142 (2) (1966) 570.

[32] M. Schumm, ZnO-based Semiconductors Studied by Raman Spectroscopy: Semimagnetic Alloying, Doping and Nanostructures, (Ph.D. thesis) JuliusMaximilians-Universität, Würzburg, Germany, 2008

[33] H. Bai, X. Liu, Green hydrothermal synthesis and photoluminescence property of ZnO 2 nanoparticles, Mater. Lett. 64 (3) (2010) 341-343.

[34] B. Cheng, Y. Xiao, G. Wu, L. Zhang, The vibrational properties of onedimensional ZnO: Ce nanostructures, Appl. Phys. Lett. 84 (3) (2004) 416-418.

[35] J. Wang, G. Huang, X. Zhong, L. Sun, Y. Zhou, E. Liu, Raman scattering and high temperature ferromagnetism of Mn-doped $\mathrm{ZnO}$ nanoparticles, Appl. Phys. Lett. 88 (25) (2006) 252502

[36] L. Yang, Y. Tang, A. Hu, X. Chen, K. Liang, L. Zhang, Raman scattering and luminescence study on arrays of $\mathrm{ZnO}$ doped with $\mathrm{Tb} 3+$, Phys. B Condens. Matter 403 (13) (2008) 2230-2234

[37] A.V. Korotcov, Y.-S. Huang, K.-K. Tiong, D.-S. Tsai, Raman scattering characterization of well-aligned $\mathrm{RuO} 2$ and $\mathrm{IrO} 2$ nanocrystals, J. Raman Spectrosc. 38 (6) (2007) 737-749.

[38] R.S. Chen, A. Korotcov, Y.-S. Huang, D.-S. Tsai, One-dimensional conductive $\mathrm{IrO} 2$ nanocrystals, Nanotechnology 17 (9) (2006) R67.

[39] R. Thapa, S. Ghosh, S. Sinthika, E.M. Kumar, N. Park, Magnetic, elastic and optical properties of zinc peroxide ( $\mathrm{ZnO} 2$ ): First principles study, J. Alloys Compd. 620 (2015) 156-163.

[40] G. Gutsev, B. Rao, P. Jena, Systematic study of oxo, peroxo, and superoxo isomers of 3d-metal dioxides and their anions, J. Phys. Chem. A 104 (51) (2000) 11961-11971.

[41] S. Yang, J.N. Kondo, K. Hayashi, M. Hirano, K. Domen, H. Hosono, Formation and desorption of oxygen species in nanoporous crystal 12CaO 7Al2O3, Chem. Mater. 16 (1) (2004) 104-110.

[42] K. Kajihara, S. Matsuishi, K. Hayashi, M. Hirano, H. Hosono, Vibrational dynamics and oxygen diffusion in a nanoporous oxide ion conductor $12 \mathrm{CaO} 7 \mathrm{Al} 2 \mathrm{O} 3$ studied by 180 labeling and micro-Raman spectroscopy, J. Phys. Chem. C 111 (40) (2007) 14855-14861.

[43] L. Friedman, I. The Hall effect in ordered and disordered systems, Philos. Mag. B 38 (5) (1978) 467-476.

[44] C. Sellmer, T. Bronger, W. Beyer, R. Carius, Anomalous Hall effect in microcrystalline Si: H films, J. Non-Cryst. Solids 358 (17) (2012) 2044-2047.

[45] N. Mott, Conduction in glasses containing transition metal ions, J. Non-Cryst. Solids 1 (1) (1968) $1-17$.

[46] J. Tauc, Optical properties and electronic structure of amorphous $\mathrm{Ge}$ and $\mathrm{Si}$, Mater. Res. Bull. 3 (1) (1968) 37-46.

[47] S. Tan, B. Chen, X. Sun, W. Fan, H.S. Kwok, X. Zhang, S. Chua, Blueshift of optical band gap in $\mathrm{ZnO}$ thin films grown by metal-organic chemicalvapor deposition, J. Appl. Phys. 98 (1) (2005) 13505

[48] J. Bruncko, A. Vincze, M. Netrvalova, P. Šutta, D. Hasko, M. Michalka, Annealing and recrystallization of amorphous $\mathrm{ZnO}$ thin films deposited under cryogenic conditions by pulsed laser deposition, Thin Solid Films 520 (2) (2011) 866-870.

[49] W. Chen, Y. Lu, M. Wang, L. Kroner, H. Paul, H.-J. Fecht, J. Bednarcik, K. Stahl, Z. Zhang, U. Wiedwald, et al., Synthesis, thermal stability and properties of $\mathrm{ZnO} 2$ nanoparticles, J. Phys. Chem. C 113 (4) (2009) 1320-1324. 